\title{
Leveraging Electronic Ticketing to Provide Personalized Navigation in a Public Transport Network
}

\author{
Ana Aguiar, Member, IEEE, Francisco Maria Cruz Nunes, Manuel João Fernandes Silva,
} Paula Alexandra Silva, and Dirk Elias

\begin{abstract}
Public transport networks (PTNs) are difficult to use when the user is unfamiliar with the area she is traveling to, as shown by a user survey that we present in this paper. This is true for both infrequent users (including visitors) and regular users who need to travel to areas with which they are not acquainted. In these situations, adequate on-trip navigation information can substantially ease the use of public transportation and be the driving factor in motivating travelers to prefer it over other modes of transportation. However, estimating the localization of a user is not trivial, although it is critical for providing relevant information. In this paper, we propose the use of an electronic ticketing infrastructure of a PTN operator for positioning within the context of the PTN to give on-trip personalized navigation cues. To our knowledge, this is an innovative contribution that has not been described or deployed, to date, elsewhere. We assess relevant design issues for a modular cost-efficient user-friendly on-trip navigation service that uses position sensors and present the details of a proof-of-concept prototype running in our laboratory. We also present and analyze the results of a user survey on the usefulness of the service and its acceptance by users.
\end{abstract}

Index Terms-Electronic ticketing, navigation, on-trip assistance, position location estimation, service-oriented architecture.

\section{INTRODUCTION}

$\mathbf{S}$ USTAINABLE urban mobility is a key factor for a citizen's quality of life, as an increasingly larger amount of the population lives in urban areas. Acknowledging this fact, the European Union has recently approved the Action Plan on Urban Mobility [1], which supports the concept of modal shift toward public transport as a means to improve environmentally friendlier and healthier cities. The integration and interoperability of different transport networks are seen in that document as a key feature for the improvement of urban mobility, together with improved travel information, and electronic ticketing using smart cards, of which radio-frequency identification (RFID) is a special case. As a consequence of the first, multimodal

Manuscript received August 24, 2010; revised April 13, 2011, July 1, 2011, and August 9, 2011; accepted August 15, 2011. Date of publication September 30, 2011; date of current version March 5, 2012. The Associate Editor for this paper was M. Brackstone.

A. Aguiar is with the Faculty of Engineering, University of Porto, Porto, Portugal, and also with the Instituto de Telecomunicações (e-mail: ana.aguiar@fe.up.pt).

F. M. Cruz Nunes, M. J. Fernandes Silva, P. A. Silva, and D. Elias are with the Fraunhofer Portugal Research Center AICOS, 4169-007 Porto, Portugal (e-mail: francisco.nunes@fraunhofer.pt; manuel.silva@fraunhofer.pt; paula.silva@fraunhofer.pt; dirk.elias@fraunhofer.pt).

Digital Object Identifier 10.1109/TITS.2011.2167612 traveling is bound to increase, and latter technologies are key for motivating the taking up of new mobility opportunities by users.

Multimodal trips can be divided into pretrip and on-trip phases [2], [3]. The first consists of trip planning, and the latter consists of the actual trip, being further divided into atstop, on-bus, and from destination to origin, according to [3]. In each phase, passengers are subject to different cognitive challenges, experience different barriers and can be assisted by different kinds of information. Regarding the pretrip phase, the significant maturity in multimodal trip planning [2] is not always reflected in the trip planning systems of PTN operators, particularly for sporadic and infrequent travelers. A recurring example is the missing integration of street addresses and points of interest (POIs) in planning, forcing users to guess as to what is the nearest station to a museum, a public office, or a doctor's office.

With respect to the on-trip phase, Rehrl et al. [2] identified other barriers of multimodal traveling that deter particularly people unfamiliar with the PTN from using public transport. Among those barriers are the complexity of PTN and the difficulty during transfers, which often lead to passengers getting lost during a modal change, as well as increased cognitive load due to the lack of integrated and personalized on-trip information.

On another field of battle, PTN operators are deploying electronic ticketing systems based on RFID technology to improve their operations and service to customers and attract more customers. Electronic ticketing systems commonly consist of a network of RFID readers placed at the entrance to a transport or at the station, be it underground, tram, bus, or train. Passengers carry tickets that are passive RFID cards and hold them to the reader at the entrance to the transport or at the station to validate their trip. The system verifies whether that RFID ticket is charged with the necessary amount of money or with the correct fare type to travel from that point. Tickets can usually be loaded with trips or a budget at public kiosks distributed in stations. The use of electronic ticketing systems in public transport networks (PTNs) is rapidly growing worldwide, with systems deployed in Shanghai, London, Helsinki, Istanbul, Moscow, Lisbon, Porto, among others, and enjoys good acceptance among users.

This paper presents a personal navigation service that leverages the RFID-based electronic ticketing infrastructure to 
address pretrip and on-trip issues. The main contributions of this paper are the following: 1) the use of the electronic ticketing infrastructure as a personalized location sensor within the PTN; 2) considerations on the design of a realistically deployable user-friendly context-aware on-trip navigation service; 3) details of a proof-of-concept prototype; and 4) a user survey on the acceptance of users and the willingness to pay for that service.

The next section describes how to leverage the electronic ticketing infrastructure of a multimodal PTN to provide user-relevant location information within a PTN. Afterward, Section III explains the service requirements that influenced system design, and Section VI explains the details of the system's software architecture. Sections VII and VIII describe relevant parts of the system, i.e., the itinerary calculation and the user interface. Finally, Section IX refers outstanding issues, and Section $\mathrm{X}$ concludes this paper and discusses future work.

\section{RELATED WORK}

RFID is commonly used for location and tracking of goods in supply chains [4], as well as indoor positioning [5], and has been previously proposed for improving the accuracy of Global Positioning System (GPS) positioning by spreading RFID tags in a city [6]. The system we have designed, which is known as Navi, has some similarities with the latter, but the novelty of our solution is leveraging the availability of an existing geographically widespread network of RFID readers, which was actually deployed for purposes other than positioning, to deploy location-based services. Moreover, the system that we propose does not provide generalized positioning but position within the context of the PTN. In addition, it delivers personalized information about the user's chosen route and not generic information about the PTN or nearby stops.

A major advantage of using electronic ticketing for positioning and Short Message Service (SMS) for providing indications is that both are independent of the capabilities of the devices owned by the passengers. This differentiates Navi from other trip planners and navigation assistants such as Navitime [7], ENOSIS [8], or OneBusAway [9]. Navitime is a multimodal navigation system available in Japan that uses GPS for outdoor positioning and cellular towers for when GPS is not available. For one, these positioning systems are not available on all mobile phones. Moreover, Navitime requires an Internet connection while on the move, which is also not available on all phones and can be costly for a large number of users, specifically roamers. ENOSIS, which is another multimodal trip companion deployed in Greece, offers a fully different set of functionalities, such as ticket booking and departure time alerts; is more complex; and involves a much wider range of stakeholders. Navi is much simpler and less costly to deploy, as it is envisioned as an enhanced service that increases the added value of an electronic ticketing infrastructure by motivating users to modal change. OneBusAway provides travel information in the Seattle area, but location awareness was just recently added as an iPhone application and provides realtime departure information about nearby stops, without any information specific to the user's route. Moreover, as opposed to Navi, NaviTime and OneBusAway target frequent users.
Another related service is Google Transit, ${ }^{1}$ which supports addresses or POIs as start and destination for over 455 cities, ${ }^{2}$ lets users choose a type of transport, and includes walking times in the calculation; however, it does not provide on-trip indications based on user location or enable integration of updated traffic information in the indications given to the user. It is also not well prepared for a multidestination itinerary, requiring the user to choose the order of places to visit. Moreover, it does not run on standard mobile devices, which are bound to be around for another couple of years.

\section{System Design REQUIREMENTS}

We designed Navi with three goals in mind, i.e., deployability in the very near future, extendibility, and usability. The first goal is a consequence of our intention to design a system that stakeholders perceive as realistic and is marketable, where cost efficiency plays a major role. The second should ensure that the limitations that may arise today as a consequence of deployability would not limit the possibilities to adapt the service to technological and policy evolution. Usability is critical for acceptance of technological assistants by users and will drive the choice and design of the use case in general, user interfaces, and interactions.

Deployability and extendability were translated to four system requirements.

1) Use commercial off-the-shelf technologies.

2) Use as much existing infrastructure as possible, limiting changes and additions to existing systems, as a means to keep deployment costs low. In addition, use as an interface a device that users carry around with them.

3) Avoid the use of mobile data connections to keep service costs low for passengers, particularly for tourists who are roaming.

4) Create a highly modular software architecture with clearly defined functionalities and well-known interfaces as a means to facilitate adaptation to different deployment scenarios and enable easy extension of parts of the system.

Usability of the system translates into the preferable use of technologies with which passengers are familiar, minimizing interaction with the user, and requesting a minimum change of his habits. Moreover, the interaction should be intuitive, and the interface should be designed so that users that are unfamiliar with the service feel comfortable using it.

\section{Navi: Context-Aware Personal Navigator}

For proof of concept, we designed Navi, which is a contextaware PTN navigator that delivers personalized information to the passenger using electronic ticketing systems as position sensor. In this section, we describe the usage scenario of the envisioned personal navigator complying with the aforementioned design requirements and targeting infrequent passengers of a PTN, which have fundamentally different needs than

\footnotetext{
${ }^{1}$ http://www.google.com/intl/en/landing/transit/\#mdy

${ }^{2}$ Web site's own statistics on Jan. 2011.
} 
frequent users [9]. In this group, we include not only tourists or visitors but frequent users as well when headed to destinations out of their current and known parts of the transport network. The service focuses on providing personalization and mobility characteristic of digital assistants, as stated by Rehrl et al. [2]. ${ }^{3}$

The service assists a passenger in the pretrip phase by offering an intuitive web-based interface to plan his trip and, thereby, gain information about the destination(s) he intends to visit in the city. Destinations here are points of touristic interest, public offices, or street addresses and will henceforth be called POIs. The web interface is available over the Internet or on kiosks that can be distributed at major PTN stations, hotels, tourist offices, etc. The system calculates the best route and sequence of POIs to visit the desired destinations considering multimodal transportation (by foot, bus, and subway), frequency of public transports, travel times between PTN stops, average waiting times for transport change, walking times to POI and back to the station, and even visiting times for touristic POI.

The on-trip assistance is triggered by the validation of the electronic ticket at each entrance to a transport or station and consists of an SMS sent to the passenger's mobile phone giving indications on how to proceed in his itinerary. To enable this, the PTN must only provide real-time connectivity of the electronic ticket readers to a back office, which then forwards necessary information to the Navi service (see Section VI-B for more details). The rest of this paper assumes that this is available and that recent developments in intelligent transport systems make it realistic to believe that; if not, the necessary connectivity will soon be added for purposes of telemetry. Alternatively, the expected adoption of near-field communication (NFC) in mobile phones, as supported by the GSM Association, will ease the use of electronic ticketing as position sensor within a PTN.

\section{USER SURVEY}

A web survey was designed to assess the difficulties that passengers experience when using public transports and explore their familiarity with the technologies that we propose for navigation support and the perceived usefulness of a PTN navigation assistant. The survey was distributed among university students and their contacts. Although the sample may cause biased results to some extent, the results give useful insight into user preferences regarding means of interaction and show that the proposed service is considered useful by more than twothirds of the respondents.

A total of 834 people answered the survey, although not all respondents answered all the questions, so percentages were calculated in relation to the total number of respondents to each question. The respondents were $64 \%$ male, $84 \%$ younger than 35 , and only $3 \%$ older than 50 . Of the respondents, $95 \%$ were Portuguese, and $98 \%$ stated that they live in Portugal. The educational level of the respondents was very high, with about $90 \%$ currently pursuing or holding a university degree.

\footnotetext{
${ }^{3} \mathrm{~A}$ video illustrating the usage scenario for the case of a tourist in Porto can be found in http://youtu.be/BRpqmFEultU. The interface has been redesigned since, as can be seen in the video attached to this submission.
}

TABLE I

Survey: "If You Ever ExperienCEd Difficulties USING PTN, What ARE THE REASONS THAT CAUSED THEM?"

\begin{tabular}{ll}
\hline Little knowledge of the area where I want to go to & $49 \%$ \\
Little knowledge of the PTN & $34 \%$ \\
Difficulties relating names in the transport service and & $27 \%$ \\
the places I want to go to & \\
$\begin{array}{l}\text { Difficulties with the information system of the trans- } \\
\text { port service (e.g.: reading, navigation, etc.) }\end{array}$ & \\
\hline
\end{tabular}

\section{A. Navigating in a PTN}

When asked about whether they could easily find their way in the PTN using the available information, $49 \%$ of the respondents who sometimes use public transport answered that they always can, 25\% said that they can usually find their way, $25 \%$ said that they can only do it in the area in which they live, and $2 \%$ answered that they are incapable of finding their way without help. Answers regarding the reasons for the difficulties experienced can be seen in Table I.

Although 49\% referred that they always easily found their way in a PTN, $62 \%$ of them admitted to having experienced difficulties sometimes when answering the question "If you ever experienced difficulties using the PTN, what are the reasons that caused them?," mostly due to lack of knowledge of the area in which they are traveling and of the PTN. This reinforces our assumption that the navigation service can be most helpful for infrequent users.

\section{B. Familiarity With Devices}

The respondents were asked about some of their habits with the Internet and mobile phone to gain insight into their familiarity with the technologies that we intended to use: web tools for planning and SMS for receiving navigation indications. When asked about the frequency of mobile phone use, 95\% answered "everyday" or "several times a day." When asked about when they last received a text message, $80 \%$ of the users answered "today," and $18 \%$ answered this "week." Considering the use of the Internet, $97 \%$ answered that they use it everyday. We also found out that more than $80 \%$ of the respondents plan their trips in advance, and the preferred planning tools are online tools, PTN timetables, PTN maps, and tourist guides.

\section{Receptiveness to the Proposed Navigation Service}

To assess the reaction of passengers to the services that we propose, we asked the respondents to grade between 0 and 4 the usefulness of navigation services, where 0 is "not useful" and 4 is "very useful." Table II shows the percentage of respondents that classified each service as little or not useful (grades 0 or 1) and the percentage that classified it as useful or very useful. The services proposed were always considered useful by more than $65 \%$ of the respondents and "not useful" by more than $5 \%$. Sending indications to the mobile phone and suggesting routes to visit a city in a given time were considered very useful by $40 \%$ of the respondents. Hence, we conclude that the services that we propose are relevant and would enjoy good acceptance. 
TABLE II

Percentage of Respondents Who Classified the Service as "LITTLE OR NOT USEFUL" AND "USEFUL OR VERY USEFUL"

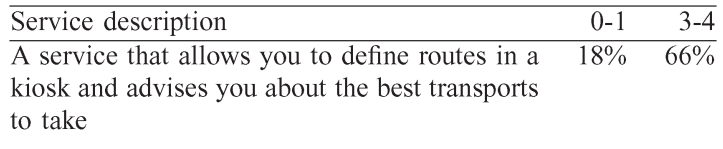

A service that notices that you are taking the wrong way, informs you and lets you know how to get back to the best route

A service that sends guidelines to your mobile phone with the best options to make your trip (e.g.: Your goal is to go to the City Hall and the Pharmacy in the morning. The system gives you the best indications, providing, when appropriate, information on the best transport, or the combination of transports to use)

A service that suggests routes to visit the city (e.g.: You are visiting a town and the system suggests you tours, parks, monuments to visit given a pre-defined tour time)

\section{NAVi SERVice AND PROTOTyPE}

We describe the two phases of a service described in Section IV as sequences of actions in the next section and continue with the details of the prototype implementation in the next section.

\section{A. Service Architecture}

The Navi service is designed as a distributed application for flexibility. All functionalities are distributed on independent modules whose functionality can be remotely accessed. In this way, the modules can be independently extended or upgraded; functionalities can be easily added and extended without interfering with existing functionalities; and ultimately, different services can be offered and maintained by different stakeholders, as is often the case with PTN operators.

The service consists of a pretrip and an on-trip phase. During the first, the service obtains the list of POIs to visit from the user and returns the ordered list corresponding to the calculated itinerary. This is associated with the card identification (ID) and phone number, which identifies the user. To achieve this, the service carries out the following sequence of actions described with pseudocode:

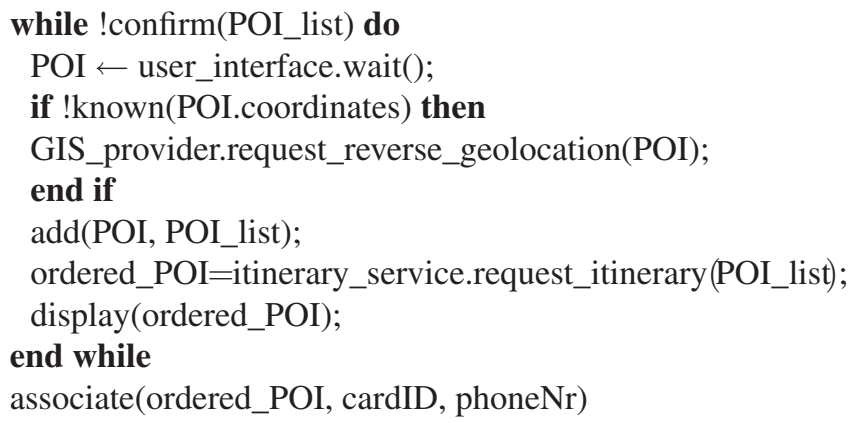

The on-trip service is triggered by the validation of an electronic ticket. Upon this trigger, the Navi service provider carries out the sequence of actions shown here in pseudocode, which culminates with sending the navigation indications to the user.

[cardID, current_station] $\leftarrow$ PTN.wait_ticket_validation(); ordered_POI $\leftarrow$ get_POI_list(cardID); unvisited_POI $\leftarrow$ estimate_and_flag_visited_POI(ordered_ POI, current_station);

ordered_remaining_POI $\leftarrow$ itinerary_service.request_ itinerary (unvisited_POI);

transports $\leftarrow$ itinerary_service.request_route(current_station, ordered_remaining_POI);

SMS_text $\leftarrow$ build_SMS(transports);

phoneNr $\leftarrow$ get_phone(cardID);

SMS_service.send(SMS_text, phoneNr).

The Navi service was designed to take into account variable schedules and travel times according to the time of day, as well as PTN anomalies, by recalculation of the ordered list of unvisited POIs and the sequence of transports to take between two stations only step by step, i.e., as the user moves along his itinerary. Hence, if any changes to the PTN transport service occur during the on-trip phase, they can be incorporated in the navigation instructions given to the user. Moreover, recalculating the itinerary for the unvisited POIs at every ticket validation enables the service to deal with the case of the passenger getting lost and showing up at a station where he is not expected. He will be given indications on how to get to his next estimated unvisited POI anyway.

To achieve this, the service estimates the POIs that the passenger has visited based on the planned itinerary before calculating the itinerary for the remaining POIs. If the user shows up at the station at which he was expected to be, the service assumes that he has been to the POIs that were on the way according to the planned itinerary. If the user shows up somewhere else, the system estimates the likely route taken from the last known POI to the current station, and the POIs on the way are marked as visited. This estimation can be erroneous for two reasons: First, the estimation that the user visited the POIs on his way from the last known POI to this station may be erroneous. This was a design decision that requires user validation during pilot tests. Second, the user may show up at an unexpected station, because he decided to change his plans. To account for this scenario, we allow the user to pause, restart, and stop the service by responding to indications with an SMS with the desired command.

Finally, SMS service does not offer service guarantees; it is possible for the SMS with instructions not to arrive in a timely fashion, i.e., it can be delayed beyond its useful lifespan within the operator's network. This risk is inherent to SMS service, and there is no preventive measure that can be taken by the service provider.

\section{B. Prototype Architecture}

The architecture of the Navi service prototype is shown in Fig. 1. The prototype consists of a kiosk with an ASUS miniPC P242, which is powered by an Intel Celeron 220 with 2-GB random-access memory, a touchscreen, and a connected 


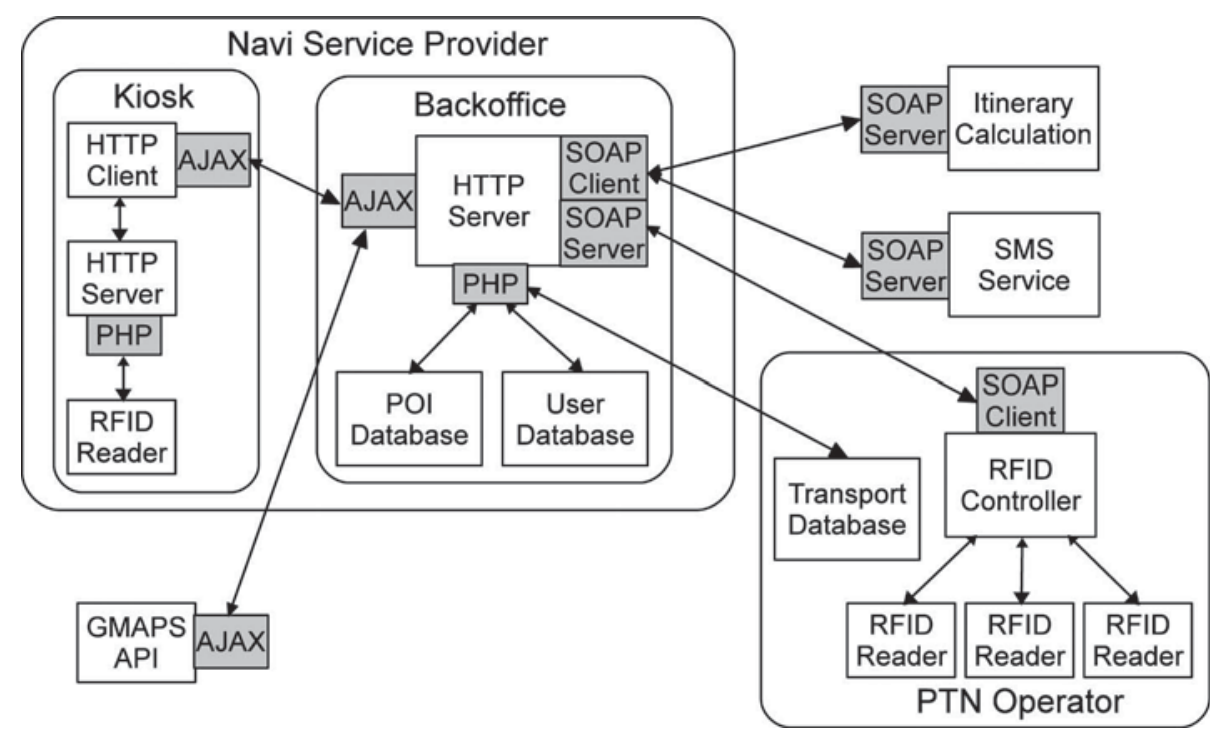

Fig. 1. Software architecture of the navigator service prototype as running in the premises of Fraunhofer Portugal AICOS.

ISO14443B-capable RFID card reader, together with a back office concurrently running on a virtual machine with nine others on a four-core 2.5-GHz processor with 1-GB memory, and six other card readers emulating PTN stations. The machines were running the Ubuntu Server Long Term Support Edition on an Apache 2 HTTP server, with PHP 5, and all databases are implemented in MySQL 5, all with default configurations, as the prototype was fully inside the premises of Fraunhofer AICOS.

The prototype implements the service described using a service-oriented architecture [10] for flexibility and platform portability. The functionalities of the independent modules are exposed through Web Services Description Language ${ }^{4}$ and accessible over Simple Object Access Protocol (SOAP). ${ }^{5}$ The route calculation was implemented in $\mathrm{C}++$ integrated with the libgsoap v2.7.

For enabling electronic ticketing as a position sensor, the PTN operator is only requested to provide real-time connectivity of the electronic ticket readers to a back office (the "RFID Controller" in Fig. 1), which notifies the Navi service through a SOAP call, indicating the ID of the card and the station where it was read as parameters. This action triggers the Navi service to send indications each time the passenger validates his ticket. ${ }^{6}$

The Navi provider's infrastructure consists of a back office that implements the workflows described in the previous section and an HTTP server hosting the user interface. The graphical user interface is available on the web and can be accessed from a kiosk, any personal computer, or even mobile devices with browsing capabilities. It was developed using XTML, JavaScript, and CSS, with AJAX for database calls and was automatically generated using PHP scripts with the help of two

\footnotetext{
${ }^{4}$ http://www.w3.org/TR/wsdl/

${ }^{5}$ http://www.w3.org/TR/soap/

${ }^{6}$ The advent of NFC will enable alternative deployment of the service, which no longer requires connectivity of the readers, and this scenario will be handled as future work.
}

PHP libraries Smarty ${ }^{7}$ and Pear. ${ }^{8}$ The first is a template engine that enables separation between logics and the interface itself. The latter enables encapsulating the database queries, easing query generation.

For trip planning as described in Section IV, the Navi service needs access to a map server, a geographic information system (GIS) database of the PTN stations and POIs, and a reverse geo-coding service to map street addresses into coordinates. For the prototype, these were obtained from the Google Maps application programming interface (API). ${ }^{9}$

Regarding the availability of the station GIS data and transport schedules, several PTN operators have made APIs available on the Internet to give access to not only transport routes and schedules but also real-time information on the transport, such as the expected arrival time at a certain stop. ${ }^{10}$ More recently, Google has provided a General Transit Feed Specification (GTFS) ${ }^{11}$ which would make all the necessary information available to the Navi service. Currently, more than 455 transit agencies and operators worldwide publish real-time data about their PTN using GTFS. Navi is currently not compatible with GTFS, because it was not available at the time the service was a prototype, but the service would require only small changes to use the GTFS specification.

The itinerary calculation service is offered through SOAP calls. (The algorithms are described in Section VII.) Itinerary calculation requires transport schedules and data from a GIS system, i.e., walking distances between points to visit and closest PTN stations, which are also obtained from the Google Maps API. The Navi back office gathers all the necessary GIS information and transforms it into a weighted graph to which the best itinerary calculation algorithms can be applied.

\footnotetext{
${ }^{7}$ http://www.smarty.net/

${ }^{8} \mathrm{http}: / /$ pear.php.net/

${ }^{9} \mathrm{http}: / /$ code.google.com/apis/maps/

${ }^{10}$ Bay Area Rapid Transit: http://bart.gov/schedules/developers/index.aspx; CTA Bus Tracker API: http://ctabusapi.jottit.com/; TriMet-Transit tracking service for Portland, OR: http://developer.trimet.org/

${ }^{11}$ http://www.google.com/intl/en/landing/transit/\#mdy
} 
Finally, kiosks, if they are part of the service, need only to run a web browser to access the interface. However, to offer the user an easy interface to associate his ticket ID with his list of POIs to visit, additional hardware and software are needed at the kiosks. We opted for the installation of an RFID card reader plus an HTTP server so that the card ID number retrieved by the driver of the RFID reader could be passed to the server using HTTP requests through a PHP script. The HTTP server running on the kiosk was required, because JavaScript does not grant access to hardware, and PHP scripts are server-side scripts. Nevertheless, the kiosk runs very few software modules so that maintenance of the distributed part of the infrastructure is limited.

\section{Multimodal Itinerary CALCUlation}

Calculation of the best route between the POIs that the user wants to visit and the best sequence in which to visit them are critical functionalities for the navigator service. Currently, itinerary services commonly require the user to list the stations of the PTN that he wants to visit and then shows the user the best path between every two of those stations. On the one hand, this is not user friendly, particularly for infrequent users, who often cannot match places they wish to go with the nearest station(s). Second, the users do not necessarily know the best sequence to visit several POIs. Thus, we take a different approach. The user inputs the destinations he wants to visit, and the system calculates the best itinerary to visit them all: the corresponding stations, the order in which they can best be visited, and the sequence of transports to take between every other one.

We designed a multimodal itinerary calculator that finds the best itinerary to visit several POIs in the network, considering both the public transports and the walking distances between the stations and the POIs to visit. We were confronted with finding algorithms that are capable of delivering solutions that are both accurate and timely, as itinerary and route calculation are embedded in the user interaction in both the pretrip and on-trip phases (see Section VI-A). In the pretrip phase, the new itinerary is calculated every time that a POI is added (or removed); in the on-trip phase, itinerary and route calculations are performed every time a ticket validation occurs. In both situations, the user expects a response within a few seconds. Since the graph of a PTN typically has a very large number of nodes, ${ }^{12}$ we separate the problem into two problems: calculation of the shortest paths between every two known nodes (PTN and known POIs) and calculation of the best itinerary to visit all the POIs that a user chooses. We will not go into the details of the mathematical modeling of the problems due to a lack of space.

1) All Pairs Shortest Paths: The stations of the PTN are nodes in a weighted graph, with edges representing the possible connections between them, whose weight is the travel time. Average travel times between nodes (stations) of the PTN can be obtained from the transport schedules.

\footnotetext{
${ }^{12}$ Porto, which is a middle-size Portuguese city, has more than 2000 bus and subway stations; London has 270 subway stations and 19700 bus stations.
}

The POIs known to the service are also mapped as nodes in the graph connected both to station nodes and among themselves by edges whose weight is the corresponding walking time, which is obtained from a GIS provider. (We used Google Maps.) The only nodes of the graph that are not known in advance are the POIs entered by the user. They are added when the user enters them and the shortest paths between them, the system POIs, and the PTN nodes must be calculated in real time in the pretrip phase.

Waiting times are added for each transport connection and calculated from the amount of transports and their frequencies between every two nodes. To account for different schedules or different travel times due to varying traffic conditions at different times of day, a basic graph can exist for each period. Anomalies in the PTN require offline recalculation of these graphs, which does not influence the response time.

The shortest path between any two nodes minimizes the sum of the average travel time and the waiting time and is calculated using a hierarchical approach, as proposed in [11]. The total graph is divided into zones, and the shortest paths between all nodes within each zone are calculated, followed by the shortest paths between the boundary nodes of all zones. Boundary nodes are all nodes that have direct connections to nodes on another zone. Since the nodes of a PTN are fixed, these optimal paths can be precalculated offline. The A* algorithm [12] is used for all best path calculations due to its improved time performance over Dijkstra, where the geographical distance to the destination is used as the heuristic.

2) All POI Pairs Shortest Paths: After the subset of nodes to be visited is known, containing both system POIs and the user-entered POIs, the shortest paths between all POIs to visit are calculated. These paths consist of one or more edges of the original graph, passing by one or more stations, with or without transport changes, so that one path can consist of more than one transport. Our algorithm takes this into account and adds a fixed waiting time each time that a transport change takes place, so that paths where a single transport is taken are preferred to paths with changes of transport. These calculations are performed online in the pretrip phase, and therefore, a branch-and-bound algorithm was added to keep response times acceptable.

3) Best Itinerary: After calculating the shortest paths between all the nodes to be visited, a new graph is built with only the nodes that the user wants to visit and edges representing the best paths between them (calculated in the previous step). The problem is now to find the best way to visit all the nodes in this new graph. We use Prim and Kurskal's algorithm to find the minimum spanning tree [12] from the start node with a branch that includes all the nodes, which is a problem that can be solved in polynomial time.

4) Response Times: Since the best paths between all PTN stations and system POIs (tourist attractions; frequent destinations such as schools, stadiums, and malls; etc.) are calculated offline, the response time does not depend 
TABLE III

MEdian AND MAXimum RESPONSE TIMES [IN SECONDS] FOR ITINERARY CALCULATION WITH AND WITHOUT BRANCH AND BOUND

\begin{tabular}{rrllll}
\hline & & \multicolumn{2}{c}{ W/O B\&B } & \multicolumn{2}{c}{ W B\&B } \\
System POI & User POI & Med & Max & Med & Max \\
\hline 5 & 2 & 1.855 & 4.78 & 1.84 & 4.85 \\
5 & 5 & 10.22 & 15.77 & 10.2 & 16.26 \\
10 & 2 & 2.33 & 3.99 & 2.34 & 4.04 \\
10 & 5 & 16.1 & 23.22 & 9.31 & 13.89 \\
15 & 2 & 13.19 & 18.77 & 4.78 & 8.94 \\
15 & 5 & 808.28 & 1363.51 & 19.31 & 116.07 \\
\hline
\end{tabular}

on the total number of nodes in the PTN. Moreover, new POIs are added in the pretrip phase so that they are already in the system for the calculations in the ontrip phase. The maximum response times for the on-trip instructions were below $0.15 \mathrm{~s}$ in all cases. Hence, the critical response time is the pretrip response time and depends on the total number of POIs to visit and mainly on the number of those that were previously unknown to the system. Response times are shown in Table III in seconds for a test PTN with six stations and a total of 27 system POIs.

The itinerary calculation algorithm keeps the median response time below $5 \mathrm{~s}$ and the maximum below $9 \mathrm{~s}$ for a total of 17 desired destinations when only two are POIs previously unknown to the system. For 15 POIs, of which five are previously unknown, the median and maximum response times rise to 10 and $14 \mathrm{~s}$, respectively. Hence, the critical factor influencing response time is the number of POIs unknown to the system, because the calculation of the all-pairs shortest paths must be done online. In these cases, we consider the response times acceptable, but it may be necessary to fine-tune the algorithm to account for more than two user POIs.

\section{USER INTERACTIONS}

The user interacts with the systems in two ways: in the pretrip phase, when he plans the trip and communicates to the service the POI he intends to visit, and in the on-trip phase, when he receives the navigation indications. The first occurs over the Internet on the user's computer or browser-enabled mobile device, or on kiosks placed at intermodal stations, service centers, or other locations in the city, such as hotels. The latter occurs per SMS, with text messages being sent by the service to the user to give him real-time indications.

\section{A. Kiosk and Web Interface}

A very simple web application is used to provide an intuitive interface that is platform independent and, thus, offers the same visual aspect on the web and kiosk. The only difference lies in the fact that the hardware available at a kiosk, i.e., an electronic card reader, cannot be assumed at home.

The kiosk has an RFID reader and a touchscreen as peripherals. The interface is developed for a touchscreen, having big buttons with clear functions consistently placed, making them easy to use even for newbies. ${ }^{13}$ Navi offers a set of predefined

\footnotetext{
${ }^{13}$ The attached video documents the use of the kiosk interface.
}

routes with different durations for visitors who want to check out the city highlights, which is a feature considered useful by $68 \%$ of the survey respondents; it also offers the possibility of adding personalized destinations. After defining the set of destinations to visit, the user is prompted for his RFID ticket card and for the phone number and receives the first indications after a final confirmation screen.

At home, the user can program a route in a similar way, but he can only associate it with his phone number. The exit screen directs him to a kiosk to associate his route with his RFID ticket. At a kiosk, the user can load his preprogrammed route by entering his phone number, eventually edit the route, and finally associate it with his electronic ticket. This functionality can be implemented on ticket vending machines available at every station.

\section{B. Navigation Instructions per SMS}

Although the ubiquitous availability of broadband wireless communications would enable a more sophisticated interface, SMS were chosen, because they are more familiar to most people and represent no additional cost even for foreigners. These facts are important, as tourists are the main target group of the system. In addition, people who are less technically skilled, less familiar with new technologies, and often do not participate in the information society today are mostly familiar with SMS.

Each time, after the system knows the route to the next destination, the SMS text containing the following necessary information to guide the user to the next ticket validation is automatically built:

1) station to get off the current transport;

2) destinations to visit by foot next to that station, if any;

3) station to take the next transport, if different from the one to get off;

4) next transport to take.

With this information, the user knows what he needs to visit the places that he desires and reach the next electronic ticket reader, which functions as the "positioning" device and triggers subsequent navigation information. As our survey shows, $88 \%$ of the users have responded to having no difficulties rereading an SMS; thus, the user can verify the indications that he has received anytime he wants at no extra costs for him or the provider.

\section{OUtstanding Issues}

There are a few open issues that we believe can be best addressed in the predeployment phase. One is the implementation of exposing the PTN electronic ticket readings, as well as the resources that are required from the PTN information system. Another related issue is the estimation of the expected request load, expected amount of users, expected number of POIs per itinerary, and frequency of those requests. These results are required to properly determine the required computing capability for the service provider. Moreover, a pilot trial with user involvement should clarify how to deal with requests that are lost or not answered on time, as well as determine acceptable response times, availability, and continuity requirements. 


\section{Conclusion And Outlook}

This paper has presented Navi, which is a novel navigator service that leverages electronic ticketing for positioning within a transport network to deliver personalized navigation indications within that network. The navigator service designed and prototyped as proof of concept is an added value service that can be deployed at low extra cost on top of an existing infrastructure, thus showing that the deployment of electronic ticketing not only improves operations but can be leveraged upon to enhance user experience. This can be crucial to motivate modal change from private transport, easing the adoption of multimodal PTN services, and, at the same time, increasing the amount of PTN passengers and enhancing the quality of life in cities. The results of the conducted user survey indicate that the service would enjoy good acceptance. The prototype's modular design enables easy deployment of a pilot trial, which should be the main drive for service enhancements.

With the advent of NFC-enabled mobile phones and ongoing field trials with NFC-based tickets stored on the user's mobile phone, the service could be redesigned to reside on an NFCenabled phone. Moreover, the service could be extended to route passengers according to the needs of the operator in case of mass events. From another point of view, PTN RFID readers could be used to improve GPS positioning on mobile phones with NFC, similarly to what was proposed in [6]. Improving real-time multimodal itinerary calculation services that consider live PTN and traffic information is another issue for further work. Finally, formal usability tests should be conducted to validate and improve the user interactions.

\section{REFERENCES}

[1] E. Commission, Action plan on urban mobility, Communication from the Commission to the European Parliament, the Council, the European Economic and Social Committee, and the Committee of the Regions, Sep. 2009

[2] K. Rehrl, S. Bruntsch, and H.-J. Mentz, "Assisting multimodal travelers: Design and prototypical implementation of a personal travel companion," IEEE Trans. Intell. Transp. Syst., vol. 8, no. 1, pp. 31-42, Mar. 2007.

[3] B. Caulfield and M. O'Mahony, "An examination of the public transport information requirements of users," IEEE Trans. Intell. Transp. Syst., vol. 8, no. 1, pp. 21-30, Mar. 2007.

[4] A. Juels, "RFID security and privacy: A research survey," J. Sel. Areas Commun., vol. 24, no. 2, pp. 381-394, Feb. 2006.

[5] R. Tesoriero, J. Gallud, M. Lozano, and V. Penichet, "Using active and passive RFID technology to support indoor location-aware systems," IEEE Trans. Consum. Electron., vol. 54, no. 2, pp. 578-583, May 2008.

[6] H. D. Chon, S. Jun, H. Jung, and S. W. An, "Using RFID for accurate positioning," J. Global Positioning Syst., vol. 3, no. 1/2, pp. 32-39, 2004.

[7] M. Arikaa, S. Konomi, and K. Ohnishi, "Navitime: Supporting pedestrian navigation in the real world," IEEE Pervasive Comput., vol. 6, no. 3, pp. 21-29, Jul.-Sep. 2007.

[8] K. G. Zografos, K. N. Androutsopoulos, and V. Spitadakis, "Design and assessment of an online passenger information system for integrated multimodal trip planning," IEEE Trans. Intell. Transp. Syst., vol. 10, no. 2, pp. 311-323, Jun. 2009.

[9] B. Ferris, K. Watkins, and A. Borning, "Location-aware tools for improving public transit usability," IEEE Pervasive Comput., vol. 9, no. 1, pp. 13-19, Jan.-Mar. 2010.

[10] M. Papazoglou, Web Services: Principles and Technology. Englewood Cliffs, NJ: Pearson Prentice-Hall, 2007.

[11] N. Jing, Y. W. Huang, and E. A. Rundensteiner, "Hierarchical optimization of optimal path finding for transportation applications," in Proc. ACM Conf. Inf. Knowl. Manage., 1996, pp. 261-268.

[12] A. Drozdek, Data Structures and Algorithms in $C++, 3 \mathrm{rd}$ ed. Boston, MA: Thomson, 2005.

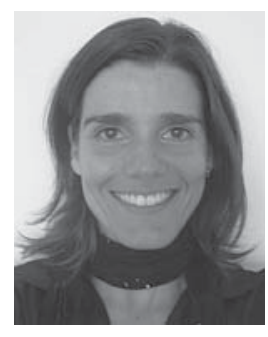

Ana Aguiar (M'93) received the M.Sc. degree in electrical and computer engineering from the University of Porto, Porto, Portugal, in 1998 and the $\mathrm{Ph} . \mathrm{D}$. degree in telecommunication networks from the Technical University of Berlin, Berlin, Germany, in 2008 .

During her Ph.D. studies, she worked on qualityof-service support for wireless networks and on measurement and prediction of wireless channels. Afterward, she worked on service-oriented architectures and wireless technologies applied to ambientassisted living at Fraunhofer Portugal AICOS. Since 2009, she has been an Assistant Professor with the Department of Electrical Engineering, University of Porto. Her research interests include distributed systems and wireless networking, particularly vehicular networks, context-aware services, and wireless channel measurement and characterization.

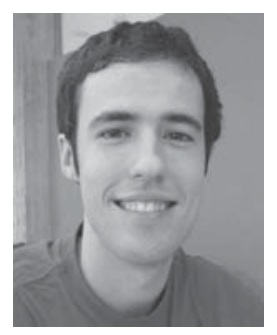

Francisco Maria Cruz Nunes received the M.Sc. degree in informatics engineering from the University of Porto, Porto, Portugal, in 2010.

$\mathrm{He}$ is currently with the Fraunhofer Portugal Research Center AICOS, Porto. His research interests include human-computer interaction with and for older adults and mobile application design.

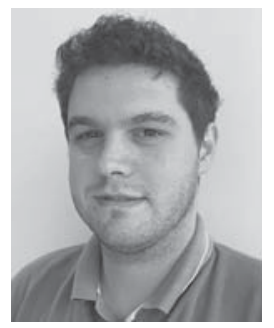

Manuel João Fernandes Silva received the M.Sc. degree in computer science from the University of Porto, Porto, Portugal.

$\mathrm{He}$ is currently with the Fraunhofer Portugal Research Center AICOS, Porto. His research interests include artificial intelligence and mobile application design.

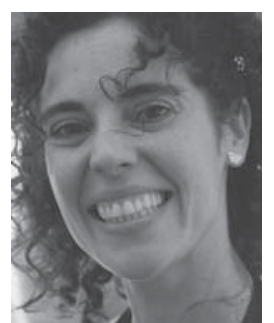

Paula Alexandra Silva received the M.S. degree in multimedia technologies from the University of Porto, Porto, Portugal, a Licentiate in new communication technologies from the University of Aveiro, Aveiro, Portugal, and the Ph.D. degree in computer science from Lancaster University, Lancaster, U.K., working in the field of human-computer interaction. Her thesis was on the BadIdeas method for supporting creativity and innovation in design.

Between 1997 and 2003, she was a University Lecturer and worked on user interface usability, analysis, design, and evaluation. Between February 2008 and June 2009, she taught design and human-computer interfaces with the University of Madeira, Funchal, Portugal. She is currently a Senior Scientist with the Fraunhofer Portugal Research Center AICOS, Porto, and a teacher with the University of Porto.

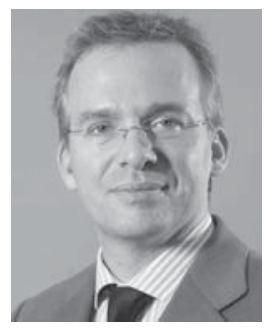

Dirk Elias received the Dipl-Ing. degree from the Technical University of Munich, Munich, Germany, in 1992 and the Ph.D. degree from the Technical University of Berlin, Berlin, Germany, in 1996, both in electrical engineering.

Between 1992 and 1996, his work was focused on multifunctional end systems with direct access to automated teller machine networks, and between 1996 and 2000, he led the Laboratory for Home and Office Technologies as Deputy Director of the Fraunhofer FOKUS Competence Center for Advanced Technologies and Systems. In 2000, he started IVISTAR AG. He is currently Head of the Executive Board of Fraunhofer Portugal, Porto, Portugal, and the Director of the Fraunhofer Portugal Research Center AICOS. Since May 2008, he has been a Full Professor with the University of Porto. 